\title{
Satisfacción Laboral y Burnout en Trabajos Poco Cualificados: Diferencias entre Sexos en Población Inmigrante
}

\section{Job Satisfaction and Burnout in Low-Skilled Jobs: Sex Differences on the Immigrant Population}

\author{
Mª Pilar Moreno-Jiménez, Mª Luisa Ríos-Rodríguez, Jesús Canto-Ortiz, Jesús San Martín-García \\ y Fabiola Perles-Nova \\ Universidad de Málaga
}

\begin{abstract}
Resumen. El objetivo de este estudio es profundizar en el análisis de la satisfacción laboral en trabajos de escasa cualificación realizados por personas inmigrantes, buscando posibles diferencias entre hombres y mujeres. Se analiza una muestra de 250 inmigrantes en España procedentes de diferentes culturas. Las variables evaluadas son: satisfacción laboral, burnout y diferentes características laborales. No se han encontrado diferencias entre hombres y mujeres respecto al burnout ni a la satisfacción laboral. Cinismo y agotamiento correlacionan negativamente con la satisfacción laboral. Los resultados del análisis de regresión muestran modelos predictivos diferentes según el sexo: en los hombres la eficacia profesional y el cinismo son más relevantes que en las mujeres, y entre ellas el agotamiento y el control estricto predicen de forma negativa la satisfacción laboral. Además, la satisfacción con el tiempo de libre y la estabilidad laboral predicen la satisfacción laboral en ambos grupos de inmigrantes.

Palabras clave: satisfacción laboral, burnout, inmigrantes, características laborales.
\end{abstract}

\begin{abstract}
The principal aim of this study tries to analyze the job satisfaction into low-skilled jobs realized by immigrants, looking for differences between women and men. Is analyzed a sample of 250 immigrants who live in Spain proceeding from different cultures. The variables evaluated across written questionnaire are: job satisfaction, burnout and some job characteristics. No differences were found between men and women in burnout or job satisfaction. So much cynicism as the depletion negatively correlated with job satisfaction. The results of the regression analysis showed predictive models differ according to sex: in men, the professional effectiveness and cynicism are more important than women; in women the exhaustion is negatively predicted job satisfaction. In addition, satisfaction with free time and job security predicts job satisfaction in both groups of immigrants.

Keywords: job satisfaction, burnout, immigrants, job characteristics.
\end{abstract}

Las migraciones son una realidad consustancial a todas las civilizaciones; las personas se trasladan de unas regiones a otras con el objetivo de vivir en mejores condiciones de vida. Y España no es ajena a esta realidad. Según los últimos datos publicados por la Secretaría de Estado de inmigración y emigración, a 30 de junio del 2010, en España hay un total de 4.744.169 de extranjeros con tarjeta de residencia en vigor, lo que supone un constante aumento respecto a hace pocos años.

En el fenómeno migratorio son muchos los factores $y$ agentes que participan, relacionados con el inmigrante, con las condiciones de su migración, con las características de la sociedad que recibe, etc. (Berry, Poortinga, Segall y Dasen, 1992; Berry, 1997). Todo ello hace que la inmigración sea un fenómeno difícil y muchas veces, problemático. Cuando dos sistemas culturales distintos interaccionan, se produce entre ellos

Enviar la correspondencia a Pilar Moreno-Jiménez. Dpto. Psicología Social, Trabajo Social, Antropología Social y Servicios Sociales. Facultad de Psicología, Universidad de Málaga. Campus Teatinos. 29071 Málaga.E-mail: mpilar@uma.es un equilibrio de fuerzas que Redfield, Linton y Herskovists (1936, citado en Bravo, 1993) denominaron aculturación. Este proceso de aculturación comprende los fenómenos que se producen cuando grupos de diferentes culturas tienen contactos y, en consecuencia, cambios en los patrones de la cultura original de uno o ambos grupos. De hecho, en el caso de la inmigración, no existe tal equilibrio entre culturas lo que supone un mayor cambio en la cultura del grupo minoritario. En general, la aculturación se refiere al aprendizaje de un nuevo repertorio conductual apropiado al nuevo contexto cultural (Berry, 1997), también se refiere al desaprendizaje de algunos aspectos previos no adecuados en el país de acogida, y a los conflictos culturales que pueden surgir.

El contraste entre la cultura del país de origen y la del país de destino supone un gran esfuerzo de adaptación, un proceso que conlleva intensos niveles de estrés. Incluso se han observado un conjunto de síntomas característicos, denominado Síndrome de Ulises (Achotegui, 2002) que sufren algunos inmigrantes. La combinación de soledad, fracaso en el logro de los objetivos, vivencia de carencias extremas y terror son 
la base psicológica y psicosocial del Síndrome del Inmigrante con Estrés Crónico y Multiple o Síndrome de Ulises. La sintomatología (Achotegui, 2004) se expresa en llantos, tristeza, culpa, ideas de muerte, tensión, preocupaciones excesivas, insomnio, cefaleas, fatiga, confusión.

El estrés por aculturación está influido por una serie de variables incluidas, entre otros, en el modelo de aculturación de Berry (1997) como la edad, género, estrategias de aculturación, actitudes de la población de acogida, locus de control, experiencias anteriores, tiempo de estancia, idioma o estrategias de afrontamiento. Sin embargo, en la actualidad la influencia concreta de cada una de ellas sigue siendo objeto de controversias. Se ha investigado la situación de regularidad o no del inmigrado (Ramos y García-Izquierdo, 2007; Vallejo, 2009), el género (Gonzáles y Landero, 2007), la edad (Farley, Galves, Dickinson y Díaz, 2005) o el tiempo que lleva en el país de acogida (Patiño y Kirchner, 2009; Ritsner y Ponizovsky, 1999).

La realidad de la inmigración en España también se manifiesta en la fuerza de trabajo. Según datos del Ministerio de Trabajo e Inmigración, en agosto de 2010 había 1.877.935 extranjeros afiliados a la Seguridad Social. Sin duda este sector es el más castigado por la crisis económica actual, lo que se refleja en su falta de crecimiento, incluso disminución en los últimos años; por otro lado, habría que añadir un grupo de población extranjera que tiene empleos sin estar dado de alta en la Seguridad Social. Una gran parte de los extranjeros, los denominados inmigrantes económicos, se trasladan de su propio país con el objeto de mejorar su calidad de vida a través de un empleo que le ofrezca esa posibilidad. El trabajador inmigrante, además de tener que adaptarse al nuevo país, se tiene que adaptar a la organización en la que se inserta laboralmente (Ramos y García-Izquierdo, 2007). Por esto, el trabajo puede suponer un incremento del habitual estrés por aculturación antes descrito.

En España las investigaciones interesadas en la inmigración se han centrado en prejuicios y actitudes, apoyo social o estrategias de aculturación. Sin embargo, son escasos los estudios que profundizan en los riesgos psicosociales en el ámbito laboral. La influencia del trabajo en el bienestar psicológico ha sido poco estudiada en población inmigrante (Aycan y Berry, 1996). Con el presente estudio se intenta aportar algunos resultados en esta área; consideramos que la población inmigrante es especialmente sensible a problemas en el ámbito laboral, especialmente los empleados en trabajos de escasa cualificación, que constituyen un colectivo con mayor grado de vulnerabilidad (Porthé, Amable y Benach, 2007) por varias razones. Muchos trabajadores inmigrantes, al insertarse en el mercado laboral, lo hacen en trabajos temporales y/o en situación irregular. Muchos están destinados a la aceptación de trabajos rechazados por autóctonos en tareas de tipo manual, sin cualificar, arriesgados, sucios y mal paga- dos. En ocasiones, tienen acceso al empleo al que concurren autóctonos y son discriminados de forma positiva al aceptar peores condiciones de trabajo. Sin embargo, sufren una discriminación negativa en las condiciones laborales, especialmente en el salario, el tipo de trabajo y las condiciones de trabajo (Solé, 2001). Como ya se apunta en un trabajo previo (Moreno-Jiménez y Ríos, en revisión), es especialmente en la fase inicial del proyecto migratorio cuando la inserción sociolaboral se encuentra dentro de las actividades de la economía sumergida y se producen algunas situaciones de abuso (excesivas horas de trabajo y falta de pago del salario convenido). Así mismo, los trabajos que realizan los inmigrantes se inscriben dentro de las actividades de los mercados de trabajo secundarios (con escasa cualificación, salarios más bajos que para los españoles y con bajo estatus social).

El trabajo proporciona, o debería proporcionar, salud física y mental, prestigio, entretenimiento, contacto social y realización personal (Gamero, 2009; Salanova, Gracia y Peiró, 1996, entre otros). Por el contrario, la insatisfacción laboral se asocia, entre otros, con síntomas de ansiedad y depresión (Newbury y Kamali, 2001) o con el absentismo laboral (Virtanen, Kimimaki, Elovainio, Batear y Ferrie, 2003). Los primeros estudios sobre satisfacción laboral se empezaron a publicar hace varias décadas (Hoppock, 1935). Pese a ello no existe unanimidad sobre su definición, ni un modelo explicativo único de dicha satisfacción. Existen definiciones que hacen referencia a la satisfacción laboral como estado emocional o respuestas afectivas (Locke, 1976). Este autor concibe la satisfacción laboral como un estado emocional positivo o placentero de la percepción subjetiva de las experiencias laborales del trabajador. Las características del propio trabajo y las de cada trabajador condicionarán esa respuesta afectiva. En España, algunos autores han profundizado en la motivación laboral (Bravo, Peiró y Rodríguez, 1996). Muñoz (1990, p. 76), después de revisar diferentes definiciones del concepto de satisfacción laboral, define este constructo como "el sentimiento de agrado o positivo que experimenta un sujeto por el hecho de realizar un trabajo que le interesa, en un ambiente que le permite estar a gusto, dentro del ámbito de una empresa u organización que le resulta atractiva y por el que recibe una serie de compensaciones psico-socio-económicas acordes con sus expectativas". En concreto, algunas definiciones consideran la satisfacción laboral como una actitud generalizada ante el trabajo (Brief, 1998; Peiró, 1984) o como la satisfacción laboral en diferentes facetas (Peiró, 1984; Warr, Cook y Wall, 1979). En general las facetas de satisfacción laboral más mencionadas se refieren a la empresa, al contenido del trabajo, salario y condiciones de trabajo (Alonso, 2008). Warr, Cook y Wall (1979) diferenciaron entre satisfacción laboral extrínseca e intrínseca. La primera se centra en la satisfacción del trabajador con aspectos relativos a la organización del trabajo, como la jornada 
laboral, la remuneración o las condiciones físicas del trabajo. La satisfacción laboral intrínseca aborda aspectos como el reconocimiento obtenido por el trabajo, responsabilidad, promoción y aspectos relativos al contenido de la tarea.

Aunque la satisfacción laboral ya ha sido relacionada con el burnout (García et al. 1993; Miller, Ellis, Zook y Lyles, 1990), el estudio de esta relación cobra especial interés en los trabajadores inmigrantes en puestos poco cualificados. En primer lugar, por el proceso de aculturación normal en esta población, caracterizado por el estrés; y en segundo lugar, porque no hemos encontrado otras investigaciones específicas centradas en la satisfacción laboral y el burnout en trabajos no cualificados realizados por inmigrantes. La mayoría de los autores consideran que una posible respuesta al estrés laboral prolongado es el burnout (Maslach y Jackson, 1986). En general, los estudios sobre los aspectos negativos de la actividad laboral definen el burnout como un estado mental, persistente, negativo y relacionado con el trabajo, que se caracteriza principalmente por agotamiento, malestar, sentimiento de escasa competencia y motivación, desarrollando actitudes disfuncionales en el trabajo (Salanova y Llorens, 2008; Schaufeli y Enzmann, 1998). Al inicio hubo consenso al considerar este síndrome compuesto por tres dimensiones: agotamiento o fatiga producida por los excesivos esfuerzos psicológicos que se realizan en el trabajo; cinismo o indiferencia y actitudes distantes respecto a los objetivos o utilidad del trabajo; y eficacia profesional o tendencia a evaluar el propio trabajo de forma positiva; el síndrome implicaría una reducción de las creencias de eficacia y de habilidad para realizar su trabajo. Posteriormente, algunos autores han utilizado sólo el agotamiento como medida del burnout (Garden, 1989) y otros consideran que las dimensiones clave son las dos primeras (Green, Walkey y Taylor, 1991). Sin embargo, muchos de los estudios siguen incluyendo los tres componentes a la hora de estudiar el burnout, sean o no, considerados parte de un mismo concepto.

En el estudio del ámbito laboral de la población inmigrante, el sexo, entre otras, es una variable a considerar, más aún cuando las ramas de actividad en las que se inserta la población extranjera varían en función del sexo. Algunos estudios encuentran diferencias en la satisfacción laboral de hombres y mujeres. Recientemente, Sánchez, Fuentes y Artacho (2007) comprueban que la mujer presenta una satisfacción laboral superior a la del hombre, lo que es apoyado por trabajos anteriores (Alonso, 2008; Clark, 1997; Sanz de Galdeano, 2001; Sloane y Williams, 2000). Sin embargo, otros autores no apoyan dicha superioridad (Forgionne y Peeters, 1982; Linz, 2003; Ward y Sloane, 2000). Ante estos resultados contradictorios nos planteamos incluir la variable sexo en el estudio de la satisfacción laboral, el burnout y otras características laborales.
Lo más novedoso del presente estudio es el objeto mismo de investigación y las principales variables unidas en un solo trabajo: población inmigrante, trabajadores en puestos de escasa cualificación, satisfacción laboral y burnout.

El objetivo principal de este estudio se ha centrado en analizar la satisfacción laboral en población inmigrante en trabajos de escasa cualificación. Este objetivo se desglosa en objetivos específicos: 1) analizar el grado de satisfacción laboral y de burnout en los participantes; 2) analizar algunas características laborales (tareas repetitivas, trabajo aburrido, inmovilidad, control estricto, falta de estabilidad, ruido y excesivas horas al día) y la satisfacción con el tiempo libre o de descanso; 3) analizar las diferencias en satisfacción laboral, burnout y características laborales entre sexos; 4) analizar las diferencias en satisfacción laboral según procedencia, situación administrativa, turno, jornada laboral, contrato y nivel formativo; 5) relacionar la satisfacción laboral y el burnout; 6) predecir la satisfacción laboral a partir de otras variables (cinismo, eficacia profesional, agotamiento, características laborales -control estricto, inmovilidad, ruido, tareas repetitivas, muchas horas al día, trabajo aburrido, falta de estabilidad- y satisfacción con el tiempo libre), tanto en hombres como en mujeres inmigrantes. Cómo hipótesis se plantean las siguientes:

H1. Se encontrarán diferencias en satisfacción laboral, burnout y características laborales entre sexos.

H2. Se encontrarán diferencias en satisfacción laboral en función de la procedencia, situación administrativa, turno, jornada laboral, contrato y nivel formativo.

H3. El burnout y la satisfacción laboral se relacionarán significativamente, se espera correlación negativa con los factores cinismo y agotamiento, y correlación positiva con la eficacia profesional.

H4. Será posible predecir la satisfacción laboral a partir de las características laborales (tareas repetitivas, trabajo aburrido, inmovilidad, control estricto, falta de estabilidad, ruido y excesivas horas al día), la satisfacción con el tiempo libre y el burnout.

\section{Método}

\section{Participantes}

La muestra analizada fue de 250 inmigrantes procedentes de América Latina (41.3\%), Europa del Este (26.3\%), el Magreb $(27.5 \%)$ y África subsahariana (4.9\%); de los cuales, el $48 \%$ son hombres y el $52 \%$ mujeres. La media de edad obtenida es de 33.43 ( $D T=$ $8.92)$ y $32.16(D T=9.16)$ para hombres y mujeres respectivamente. Las características de la muestra reflejan 
que los participantes poseen un nivel formativo de secundaria $(45.6 \%)$ o formación universitaria $(32.8 \%)$. Se encuentran en situación administrativa regularizada un $74.1 \%$ de la muestra. Respecto a la situación laboral, en la Tabla 1 se muestra la jornada de trabajo de los participantes, el porcentaje de trabajadores en horario nocturno, así como el porcentaje de personas que realizan horas extras y que tienen contrato laboral. Todos los participantes están trabajando en empleos no cualificados: peón de construcción, servicio doméstico y cuidado de personas o en hostelería (limpieza o ayudante de cocina).

Debe señalarse, además, que el $32.9 \%$ poseen un horario de trabajo que fluctúa cada día y el $29.6 \%$ no recibe el mismo sueldo todos los meses.

Tabla 1. Porcentajes de hombres y mujeres: jornada de trabajo, turno nocturno, horas extra y contratación

\begin{tabular}{lccc}
\hline & & Hombres & Mujeres \\
\hline Jornada de trabajo & Seguida & $63.6 \%$ & $55.1 \%$ \\
Turno Nocturno & Sí & $39.2 \%$ & $23.9 \%$ \\
Horas extra & Sí & $56.2 \%$ & $48.1 \%$ \\
Remuneración de horas extra & Sí & $56.2 \%$ & $34.3 \%$ \\
Contratación & Sí & $35.7 \%$ & $40.4 \%$ \\
\hline
\end{tabular}

\section{Instrumentos}

Para recoger información sobre los diferentes aspectos sociodemográficos de los participantes, se ha elaborado una entrevista estructurada, con respuestas cerradas sobre datos demográficos (datos personales, académicos y laborales), datos relacionados con la inmigración (procedencia y situación administrativa) y datos del área social (estado civil, hijos).

El resto de variables son continuas: burnout, satisfacción laboral y características laborales. Todas estas pruebas utilizadas tienen una escala de respuesta de varios puntos, que oscilan desde el punto mínimo (nada de acuerdo, nunca) al máximo (totalmente de acuerdo, siempre).

\section{Satisfacción laboral}

Se ha utilizado la Escala de Satisfacción Laboral elaborada por Warr, Cook y Wall, (1979), NTP 394, formada por dos subescalas: subescala de satisfacción laboral intrínseca y subescala de satisfacción laboral extrínseca. La satisfacción total sería la suma de ambas subescalas, esto es, de los resultados en todos los ítems. Tras comprobar la alta correlación entre las subescalas y la existencia de un solo factor, consideramos una única variable: la satisfacción laboral. El valor de la respuesta de cada ítem oscila de 1 "muy insatisfecho" a 7 "muy satisfecho". En el presente trabajo se obtiene un alfa de Cronbach de .90 para la escala total.

\section{Burnout}

Se ha utilizado una versión adaptada al castellano del Maslach Burnout Invenntory-General Survey (MBI-GS; Maslach, Jackson y Leiter, 1996). Esta versión (Gil Monte, 2002) consta de 16 ítems que se distribuyen en tres subescalas denominadas eficacia profesional, agotamiento y cinismo. La respuesta oscila de 0 "Nunca" a 6 "todos los días". El alfa de Cronbach obtenido en este trabajo es de $.79, .84$ y .86 en las subescalas eficacia profesional, agotamiento emocional y cinismo, respectivamente.

\section{Características laborales}

Para evaluar algunos aspectos del contenido y condiciones del trabajo así como de las condiciones del empleo, se han incluido siete ítems, planteando el siguiente enunciado: "Consideras que tu trabajo tiene las siguientes características..." (Control estricto, inmovilidad, ruido, tareas repetitivas, muchas horas al día, trabajo aburrido, falta de estabilidad). La respuesta oscila de 1 "nada" a 5 "mucho". El análisis factorial realizado muestra que esta escala tiene un solo factor que explica el $43.2 \%$ de la varianza. Esta escala tiene un alpha de Cronbach de .73.

La satisfacción con la cantidad de tiempo de descanso que le permite su trabajo -tiempo libre- se evalúa con un ítem: "Tu trabajo te permite estar satisfecho con el tiempo libre". La respuesta oscila de 1 "nada" a 5 "mucho".

\section{Procedimiento}

Dos entrevistadoras realizaron la entrega, explicación y recogida de los cuestionarios. Estos se han respondido de forma individual en un tiempo aproximado de 15 minutos. Los participantes se han seleccionado teniendo en cuenta el sexo para balancear la muestra. Para la recogida de datos se utilizó la técnica de la bola de nieve (Taylor y Bogdan, 1986) y se visitaron lugares de ocio, locutorios y asociaciones de inmigrantes. Los requisitos para participar en el estudio han sido ser inmigrante en la ciudad de Málaga y estar trabajando en el momento de la recogida de datos en un puesto no cualificado (peón de construcción, servicio doméstico y cuidado de personas, hostelería -limpieza o ayudante de cocina-). Los cuestionarios eran anónimos.

\section{Resultados}

Los datos han sido analizados mediante el paquete estadístico SPSS 15.0. Para lograr los objetivos descriptivos y comparativos se utilizan medidas de tendencia central, técnicas T de Student y ANOVAS uni- 
Tabla 2. Medias y desviaciones típicas en las escalas de satisfacción laboral, burnout y características laborales en hombres y mujeres inmigrantes. Diferencias entre sexos, ANOVA utilizando puntuaciones $\mathrm{z}$

\begin{tabular}{|c|c|c|c|c|c|c|c|c|}
\hline & \multicolumn{3}{|c|}{ Hombres } & \multicolumn{3}{|c|}{ Mujeres } & \multirow[b]{2}{*}{$\mathrm{F}$} & \multirow[b]{2}{*}{$P$} \\
\hline & $\mathrm{N}$ & M & DT & $\mathrm{N}$ & M & DT & & \\
\hline SL (15-105) & 120 & 69.29 & 16.75 & 109 & 68.13 & 17.86 & .228 & .634 \\
\hline Agotamiento (1-7) & 115 & 2.91 & 1.59 & 110 & 2.92 & 1.65 & .003 & .959 \\
\hline Cinismo (1-7) & 115 & 2.62 & 1.42 & 112 & 2.51 & 1.47 & .304 & .582 \\
\hline Eficacia profesional (1-7) & 114 & 4.40 & 1.46 & 110 & 4.51 & 1.65 & .308 & .579 \\
\hline Control estricto (1-5) & 114 & 2.71 & 1.45 & 115 & 2.61 & 1.45 & .293 & .589 \\
\hline Inmovilidad (1-5) & 116 & 3.06 & 1.62 & 115 & 2.85 & 1.59 & 1.010 & .316 \\
\hline Ruido (1-5) & 122 & 3.07 & 1.61 & 114 & 2.45 & 1.42 & 9.329 & .003 \\
\hline Tareas repetitivas (1-5) & 123 & 3.53 & 1.47 & 115 & 3.69 & 1.35 & .707 & .402 \\
\hline Muchas horas al día (1-5) & 122 & 3.34 & 1.64 & 110 & 2.92 & 1.44 & 4.007 & .047 \\
\hline Trabajo aburrido (1-5) & 120 & 2.66 & 1.49 & 109 & 2.44 & 1.45 & 1.289 & .257 \\
\hline Falta de estabilidad (1-5) & 119 & 2.86 & 1.50 & 109 & 2.69 & 1.50 & .707 & .401 \\
\hline Satisfacción con lacantidad de tiempo de descanso (1-5) & 124 & 3.01 & 1.55 & 113 & 3.07 & 1.48 & .094 & .759 \\
\hline
\end{tabular}

variadas. Para las correlaciones se utiliza el coeficiente de Pearson. Para analizar la predicción de la satisfacción laboral se ha utilizado análisis de regresión, varimax, método de pasos sucesivos. Puesto que las escalas de medida poseen diferentes rangos, se utilizarán las puntuaciones $\mathrm{z}$, es decir, los valores tipificados.

En relación con los objetivos 1, 2, 3 y 4, las puntuaciones obtenidas en las diferentes variables en el grupo de hombres y de mujeres se muestran en la tabla 2. En ella se muestran las medias y desviaciones típicas obtenidas en el total de la escala de satisfacción laboral, en agotamiento, cinismo y eficacia profesional, así como las puntuaciones obtenidas en la percepción de las características laborales y la satisfacción con el tiempo de descanso en hombres y mujeres. Al analizar si existen diferencias según el sexo en las características evaluadas, se encuentran diferencias en la percepción de ruido, siendo mayor en los puestos de trabajo del grupo de hombres $(p=.003)$ y en las horas trabajadas al día, más en los hombres $(p=.047)$.

Asímismo, se comprueba si la percepción de la satisfacción en el trabajo puede ser diferente según el país de procedencia, situación administrativa (regularizados vs. no regularizados), el turno (nocturno vs. diurno -mañana o tarde-), jornada, estar contratado, y según el nivel formativo. Al realizar la prueba estadística $\mathrm{T}$ para muestras independientes, no se encuentran diferencias significativas en cada ítem de la escala de satisfacción laboral, ni en el total de la escala de satisfacción laboral en ninguno de los grupos según las diferentes condiciones.

Por lo que respecta al objetivo 5 (Relación entre burnout y satisfacción laboral), en la tabla 3 se incluyen las correlaciones entre todas las variables consideradas: satisfacción laboral, cinismo, eficacia profesional, agotamiento, características laborales (control estricto, inmovilidad, ruido, tareas repetitivas, muchas horas al día, trabajo aburrido, falta de estabi- lidad) y satisfacción con el tiempo libre. Entre las dimensiones agotamiento, cinismo y eficacia profesional sólo correlacionan significativamente cinismo $\mathrm{y}$ agotamiento en ambos grupos $(p<.001)$. Las correlaciones entre la satisfacción laboral y la eficacia son positivas, mientras que entre satisfacción laboral y las otras dos dimensiones del burnout las correlaciones son significativamente negativas en hombres y en mujeres. Destaca la alta correlación entre cinismo y agotamiento en mujeres. Además, se aprecia que la satisfacción laboral en mujeres correlaciona negativamente con la inmovilidad, muchas horas al día, tareas repetitivas y falta de estabilidad; así mismo, correlaciona positivamente con la satisfacción con el tiempo libre. En hombres, la satisfacción laboral correlaciona negativamente el ruido, muchas horas al día, trabajo aburrido y falta de estabilidad; también correlaciona positivamente con la satisfacción con el tiempo libre.

En relación con el objetivo 6 (Predicción de la satisfacción a partir de otras variables, tanto en hombres como en mujeres inmigrantes) y con el objeto de conocer si la percepción de algunas características laborales, el cinismo, el agotamiento y la eficacia profesional percibida predicen la satisfacción laboral, hemos realizado un análisis de regresión.

Aunque no existen diferencias estadísticamente significativas en los niveles de satisfacción laboral en función del sexo, se espera que la predicción de dicha satisfacción pueda ser diferente para hombres y mujeres. Los resultados del análisis de regresión (Tabla 4) muestran efectivamente modelos predictivos diferentes según el sexo: en los hombres la eficacia profesional y el cinismo son más relevantes que en las mujeres, y entre ellas el agotamiento y el control estricto predice de forma negativa la satisfacción laboral. Además, la satisfacción con el tiempo de libre y la falta de estabilidad predice la satisfacción laboral en ambos grupos de inmigrantes. 
Tabla 3. Correlaciones entre la satisfacción laboral y otras variables (cinismo, eficacia profesional, agotamiento, características laborales -control estricto, inmovilidad, ruido, tareas repetitivas, muchas horas al día, trabajo aburrido, falta de estabilidad- y satisfacción con el tiempo libre) en hombres y mujeres. Correlación de Pearson con puntuaciones z

\begin{tabular}{|c|c|c|c|c|c|c|c|c|c|c|c|c|}
\hline & 1 & 2 & 3 & 4 & 5 & 6 & 7 & 8 & 9 & 10 & 11 & 12 \\
\hline 1 & & $.201 * *$ & $.225 *$ & .111 & .003 & .110 & .174 & .097 & .040 & -.036 & -.007 & -.188 \\
\hline 2 & $.349 * *$ & & $.397 * *$ & $.371 * *$ & $.319 * *$ & $.366 * *$ & $.410 * *$ & -.069 & $.267 * *$ & -.141 & $.269 * *$ & $-.206^{*}$ \\
\hline 3 & $.298 * *$ & $.441 * *$ & & $.374 * *$ & $.283 * *$ & $.372 * *$ & $.252 * *$ & -.128 & $.264 * *$ & -.079 & .182 & -.101 \\
\hline 4 & .154 & $.405 * *$ & $.493 * *$ & & $.372 * *$ & $.452 * *$ & $.358 * *$ & -.074 & $.367 * *$ & -.141 & $.261 *$ & -.185 \\
\hline 5 & .081 & .184 & $.291 * *$ & .182 & & $.359 * *$ & $.307 * *$ & $-.306 * *$ & $.425 * *$ & -.117 & $.354 * *$ & $-.284 * *$ \\
\hline 6 & .004 & $.274 * *$ & $.343 * *$ & $.460 * *$ & $.415 * *$ & & $.538 * *$ & -.172 & $.447 * *$ & $-.332 * *$ & $.474 * *$ & $-.393 * *$ \\
\hline 7 & -.027 & .100 & $.154 * *$ & $.245^{*}$ & $.339 * *$ & $.377 * *$ & & -.169 & $.421 * *$ & -.192 & $.346 * *$ & $-.439 * *$ \\
\hline 8 & .031 & -.079 & $-.262 * *$ & -.095 & $-.362 * *$ & $-.223^{*}$ & -.138 & & $-.401 * *$ & $.234 *$ & $-.287 * *$ & $.385 * *$ \\
\hline 9 & .071 & $.308^{* *}$ & $.426 * *$ & $.307 * *$ & $.414 * *$ & $.472 * *$ & $.338 * *$ & $-.372 * *$ & & -.191 & $.706^{* *}$ & $-.519 * *$ \\
\hline 10 & .071 & -.008 & $-.136-$ & .092 & $-.244 *$ & $-.339 * *$ & -.181 & .192 & -.031 & & -.181 & $.338 * *$ \\
\hline 11 & -.006 & $.198 *$ & $.416^{* *}$ & $.326 * *$ & $.259 * *$ & $.530 * *$ & $.380 * *$ & -.166 & $.567 * *$ & -.222 & & $-.378 * *$ \\
\hline 12 & -.002 & -.082 & $-.342 * *$ & -.128 & $-.372 * *$ & $-.430 * *$ & $-.422 * *$ & $.487 * *$ & $-.308 * *$ & $.408 * *$ & -.440 & \\
\hline
\end{tabular}

Encima de la diagonal se han escrito las correlaciones en el grupo de mujeres, debajo de la diagonal para el grupo de los hombres. 1 . Control estricto; 2 . Inmovilidad; 3 . Ruido; 4 . Tareas repetitivas: 5. Muchas horas al día; 6. Trabajo aburrido; 7. Falta de estabilidad; 8. Satisfacción con la cantidad de tiempo de descanso: 9 . Agotamiento; 10. Eficacia profesional; 11. Cinismo; 12. S. Laboral. N varía de 96 a 119.

$* * p<.001 ; * p<.050$

Tabla 4. Predicción de la variable dependiente: Satisfacción laboral. Modelos para hombres y mujeres. Análisis de regresión utilizando puntuaciones z

\begin{tabular}{|c|c|c|c|c|c|c|c|}
\hline & \multicolumn{2}{|c|}{ Hombres } & \multirow[b]{2}{*}{$\mathrm{F}$} & & \multicolumn{2}{|c|}{ Mujeres } & \multirow[b]{2}{*}{$\mathrm{F}$} \\
\hline & Beta & $R^{2}$ corregida & & & Beta & $R^{2}$ corregida & \\
\hline Satisfacción tiempo de ocio & .508 & .248 & 27.416 & Agotamiento & -.550 & .293 & 33.809 \\
\hline Falta de estabilidad & -.335 & .351 & 22.668 & Falta de estabilidad & -.248 & .335 & 20.871 \\
\hline Eficacia profesional & .286 & .422 & 20.472 & Satisfacción tiempo de ocio & .211 & .364 & 16.080 \\
\hline Cinismo & -.192 & .445 & 17.042 & Control estricto & -.187 & .391 & 13.694 \\
\hline
\end{tabular}

\section{Discusión}

El objetivo principal de este estudio se ha centrado en profundizar en el análisis de la satisfacción laboral en trabajos de escasa cualificación realizados por personas inmigrantes, buscando posibles diferencias entre hombres y mujeres. Se han tenido en cuenta otros factores encontrados en diversas investigaciones: burnout (García et al., 1993; Millar et al. 1990); características de la tarea y condiciones físicas del trabajo (Renaud, 2002; Topa, Fernández y Lisbona, 2005), permiso laboral (Rinken, 2008). Esto se ha ampliado con la inclusión de otras variables que hemos considerado relevantes en esta población, como la satisfacción con el tiempo libre que cada persona posee.

Respecto a los niveles obtenidos en las diferentes variables, los datos muestran valores medios. Entre las dimensiones del burnout destaca el valor de la eficacia profesional con 4.40 en hombres y 4.51 en mujeres, en una escala de 1 a 7 . Entre las características laborales, los niveles mayores se obtienen en ítems sobre "tareas repetitivas" y "muchas horas al día". Los datos muestran que estos trabajadores inmigrantes en empleos no cualificados poseen un nivel formativo medio-alto (estudios secundarios y/o uni- versitarios, 78.4\%). Buena parte de ellos no están regularizados legalmente $(25.9 \%)$; realizan turnos nocturnos (casi el $40 \%$ de los hombres y el $24 \%$ de las mujeres); el $14 \%$ de las mujeres no cobran las horas extras que realizan y sólo una parte de estos empleados tiene contrato laboral (35.7\% de hombres y $40.4 \%$ de mujeres).

Respecto al objetivo de analizar las posibles diferencias entre hombres y mujeres (objetivo 3), sorprende que la hipótesis de partida no se cumpla. Es decir, no se han encontrado diferencias según el sexo ni en satisfacción laboral ni en burnout. Nuestros resultados coinciden con las investigaciones en las que no se encuentran diferencias en la satisfacción laboral de trabajadores o trabajadoras (Forgionne y Peeters, 1982; Gamero, 2004; García et al., 1993; Linz, 2003; Oshagbemi, 2000; Ward y Sloane, 2000). En el presente estudio, con una muestra de trabajadores inmigrantes en puestos laborales no cualificados, se puede inferir que las mujeres poseen empleos en peores condiciones, lo que las iguala en satisfacción laboral a los hombres, poseen una satisfacción laboral similar en puestos laborales diferentes a sus compañeros varones.

Dentro de este tercer objetivo se ha analizado la diferencia en satisfacción laboral para otras variables 
(procedencia, situación administrativa, turno, jornada laboral, contrato, nivel formativo). Así mismo, sorprende que por ejemplo el estatus legal (tener o no permiso de residencia) no introduce diferencias significativas en la satisfacción laboral; en el mismo sentido los trabajos de Patiño y Kirchner (2009) muestran que ese estatus no influye en el estrés ni en la calidad de vida.

La sociedad contemporánea se ha transformado en una sociedad laboral (Claes, 1987; Ovejero, 2006), donde la necesidad de trabajar se ha convertido en el deseo de trabajar. Sin embargo, el empleo remunerado no sólo desempeña un papel económico para las personas, sino que cumple una serie de funciones psicosociales tales como la autorrealización del sujeto, proporciona prestigio y estatus social y es una fuente de identidad personal (Salanova, Prieto y Peiró, 1993; Ovejero, 2006). Si el empleo no cumple estas funciones, puede producir una disminución de la satisfacción laboral o bien provocar mayor nivel del factor cinismo (burnout). Incluso en empleos sin contrato, inmigrantes sin documentación o con turno nocturno, hemos encontrado que se mantiene similar el nivel de satisfacción laboral. La satisfacción laboral puede ser producida por la preferencia del trabajador por el empleo que actualmente ocupa, respecto a otro que se imagina. Ese empleo "ideal" se construye por las experiencias propias, las observadas en otros individuos y las expectativas creadas en torno al empleo. Esto implica la existencia de correlatos entre el afecto generado por la comparación social ascendente o descendente y el burnout y la satisfacción laboral (López-Araujo, Osca y Peiró, 2007). Una posible explicación al mantenimiento de la satisfacción laboral podría ser que el grupo de referencia del inmigrante es su grupo de procedencia en el país de acogida, es decir, otros inmigrantes trabajadores que se encuentran en situaciones similares o, incluso, en desempleo. El objeto de comparación de su situación es el empleo de sus compatriotas y no tanto el de los autóctonos. En los casos en que la situación laboral en el país de origen era de mayor estatus social, se asume el descenso en dicho estatus como el precio a pagar por mejorar el nivel de vida general en el país de acogida. En la mayoría de los casos la necesidad de empleo les hace trabajar en lo que se les ofrece y de forma progresiva buscar la mejora de sus condiciones laborales y sociales.

En cuanto al objetivo quinto (relacionar satisfacción laboral y burnout), nuestra hipótesis de partida se confirma, ya que el cinismo y el agotamiento correlacionan negativamente con la satisfacción laboral; y la eficacia profesional correlaciona positivamente con esta satisfacción. Destacan las correlaciones positivas entre cinismo y agotamiento, que además, es mayor en mujeres. Estos datos coinciden con los de García et al. (1993) que también encontró correlaciones negativas y significativas entre satisfacción laboral y burnout.

El último objetivo de este trabajo ha sido conocer si algunas características laborales y el burnout predicen la satisfacción laboral en población inmigrante. Cuando nos planteamos si la predicción de la satisfacción podría ser diferente según el sexo, encontramos que una variable predice los dos modelos de satisfacción laboral tanto en hombres como en mujeres: la satisfacción con la cantidad de tiempo libre. De hecho los informes de las personas sobre las actividades que realizan en su vida diaria y cuánto tiempo invierten en esas actividades indican las preferencias, valores, estilos de vida, estado subjetivo de bienestar y satisfacción, (Sánchez y López, 1997; Sánchez-López y Aparicio, 2001). Los trabajos en este campo diferencian entre el trabajo pagado (tiempo de contrato o contratado) que es el tiempo dedicado al empleo, desplazamientos al trabajo, las pausas que se hagan en el mismo, y el trabajo no pagado (tiempo comprometido) que refleja el carácter obligatorio de tareas que no están relacionadas con el empleo: cuidado de los hijos, preparación de comida, limpieza de la casa, mantenimiento del hogar y del coche, reparaciones y compras de primera necesidad. Sánchez-Herrero (2008) presenta un análisis desde la perspectiva de sexo de la psicología del ocio en el que enfatiza el mayor tiempo dedicado por las mujeres al tiempo comprometido. En esta línea, Cruz, Noriega y Garduño (2003) señalan que cuando la estrategia para enfrentar el empobrecimiento es la integración de las mujeres al mercado de trabajo se hacen arreglos en la composición familiar que modifican la distribución de las tareas en el hogar que condicionan la utilización del tiempo libre, generando con ello un modelo de actividades recreativas, donde los varones disponen de mayor tiempo libre que las mujeres. Éstas emplean generalmente su tiempo, fuera del trabajo asalariado, en quehaceres domésticos y en el cuidado de los hijos.

Antes de concluir con este punto, es necesario resaltar la importancia que parece tener la satisfacción con el tiempo libre sobre la satisfacción laboral, ya que como se constata en diferentes trabajos, existe una fuerte relación entre ambas variables (Haworth, 1997; Haworth y Veal, 2004). La satisfacción con el ocio genera toda una serie de beneficios (mejora el humor, la felicidad, la salud física y mental...) que facilita un mejor rendimiento laboral y aumento de la satisfacción con el mismo.

En segundo lugar, la importancia de fomentar la satisfacción con el tiempo libre, se ve reforzada por tratarse de una muestra de inmigrantes, ya que en este caso también existen evidencias de cómo el uso del tiempo libre se relaciona con el proceso de aculturación en la nueva cultura (Christenson, Zabriskie, Eggett y Freeman, 2006; Stack y Iwasaki, 2009), variable ésta última que también podría estar relacionada con la satisfacción laboral.

Otra variable que predice los dos modelos predictivos planteados es la percepción de estabilidad laboral. En este sentido, Pumares-Fernández, García y Asensio (2006) conciben la búsqueda de movilidad ocupacio- 
nal como el principal motor de la movilidad geográfica de los inmigrantes después de su llegada a España, ya que éstos se desplazan desde las zonas que registran condiciones laborales más precarias hacia aquellas en las que esperan tener mejores opciones de promoción laboral. A su vez, se confirma empíricamente que la falta de estabilidad en el empleo impacta negativamente sobre la satisfacción laboral de un trabajador (Álvarez y Miles, 2006), y que los contratos laborales temporales se asocian a niveles menores de satisfacción laboral que los permanentes, fundamentalmente en varones. En la población inmigrante esta asociación se comporta de forma diferente ya que, a pesar de que la falta de estabilidad se relaciona negativamente con la satisfacción laboral, no hemos comparado los que tienen empleo estable con los de empleo poco estable, sino los que poseen contrato y los que no. Por lo tanto, puede darse la paradoja de que los trabajadores con contrato tengan empleo por poco tiempo (por ejemplo un hombre empleado en la construcción) y los no contratados posean una mayor estabilidad (por ejemplo, una mujer cuidadora de menores).

Por último, centrándonos en las diferentes variables que predicen la satisfacción laboral en mujeres, encontramos que el control estricto de las tareas predice negativamente la satisfacción laboral. Este dato no aparece en hombres. Consideramos que puede ser causado por el tipo de empleo, ya que la mujer inmigrante se dedica principalmente al trabajo doméstico o al cuidado de personas, sin compañeros de trabajo, siendo mayor la supervisión y el contacto entre empleadores y empleados.

Así mismo, el agotamiento predice más del $29 \%$ de la satisfacción laboral en las mujeres, lo que se relaciona directamente con todo lo comentado más arriba respecto a la satisfacción con el tiempo libre y la difícil conciliación entre trabajo y vida familiar. Podemos interpretar que para las mujeres inmigrantes es importante no estar agotadas o fatigadas para poder disfrutar (satisfacerse) del trabajo.

Como limitación de este trabajo señalamos que se trata de un diseño transversal, por lo que no se puede observar la evolución del burnout o de la satisfacción laboral a través del tiempo. Tal vez, otra limitación que se denota en este trabajo se refiere a las características de la muestra. Se han realizado los análisis considerando un solo grupo de trabajadores, que tienen en común el hecho de ser inmigrantes, sin embargo, poseen diferentes empleos, aunque todos de baja cualificación. Sería interesante analizar en un mismo sector laboral las mismas variables presentadas en esta investigación, o comparar los resultados entre grupos de inmigrantes trabajadores, diferenciados según sectores laborales de diferente cualificación.

A pesar de estas limitaciones, hay muy pocos estudios centrados en la satisfacción laboral o el burnout en población inmigrante. Además la inclusión de la satisfacción con el tiempo libre parece ser adecuada a la luz de los resultados y confirma la importancia creciente que posee el tiempo libre y un uso que mejore la satisfacción laboral y la satisfacción con la vida en general, lo que debe tenerse en cuenta en las intervenciones psicosociales en este ámbito.

Los resultados nos señalan además que pese a que este colectivo se encuentra en situaciones precarias de empleo, a veces se desarrolla cierto conformismo y sumisión a las circunstancias laborales (Prada, Actis y Pereda, 2000) que en nuestro caso se ha evidenciado en niveles similares en las satisfacciones laborales para situaciones diferentes (tener contrato o no tenerlo, por ejemplo). La explicación a esta realidad se encuentra, tal vez, en la identificación con el rol de "extranjero" y, por tanto, de subordinado a los intereses de las organizaciones y de las empresas. Una explicación alternativa puede encontrarse en el desconocimiento de los mecanismos para reclamar unas condiciones dignas de empleo y el temor a empeorar su realidad laboral y vital, especialmente si el trabajador está en situación administrativa no regularizada. En este sentido, sería interesante profundizar, con metodología cualitativa, en las causas de la aceptación de condiciones de empleo desfavorables sin consecuencias para la satisfacción laboral, así como explorar el papel del tiempo libre en la satisfacción según el sexo. En una sociedad cada vez más cambiante el reto está en aprender a equilibrar la responsabilidad social -atención a la diversidad culturalcon la eficiencia económica.

\section{Extended Summary}

\section{Introduction}

The influence of work on psychological well being has been little studied in the immigrant population. The present study attempts to provide some results in this area, we believe that the immigrant population is particularly sensitive to problems in the workplace, especially those employed in unskilled jobs, which constitute a group with higher degree of vulnerability (Porth, Amable \& Benach, 2007).

The job dissatisfaction is associated, among oth- ers, with symptoms of anxiety and depression (Newbury \& Kamali, 2001) or absenteeism (Virtanen, Kimimaki, Elovainio, Batear \& Ferrie, 2003). Although job satisfaction has been linked to burnout (Garcia et al. 1993; Miller, Ellis, Zook, \& Lyles, 1990), this relationship is of particular interest in migrant workers in unskilled jobs. Most authors consider that a possible response to prolonged stress is burnout (Maslach \& Jackson, 1986). In the workplace study of the immigrant population, sex is a variable to consider. Most recently, Sánchez, Fuentes 
\& Artacho (2007) find that a woman has a higher job satisfaction than men's. However, other authors do not support the superiority (Forgionne \& Peeters, 1982; Linz, 2003; Ward \& Sloane, 2000). Given these conflicting results include the sex variable in this study. The most novel aspect of this research is the object of study and major descriptors: population immigrant workers in unskilled jobs, job satisfaction and burnout.

The main objective has focused on job satisfaction among the immigrant population in low-skilled jobs. This goal is broken down into specific objectives: 1) analyze the degree of job satisfaction and burnout in participants, 2) analyze some job characteristics (repetitive tasks, boring work, immobility, strict control, lack of stability, noise and excessive hours a day) and satisfaction with leisure time; 3) analyze the differences between the sexes in job satisfaction, burnout and features labor; 4) analyze the differences in job satisfaction for other variables (origin, administrative status, shift, working hours, contract, education level); 5) relate the job satisfaction and burnout; 6) predict job satisfaction from other variables (burnout and job characteristics) both men and women immigrants.

We hypothesize: 1) Differences were found between men and women in job satisfaction, burnout and the job characteristics; 2) Differences in job satisfaction by origin, administrative status, shift, working hours, contract and level of training; 3) The burnout and job satisfaction is significantly related; 4) will be possible to predict job satisfaction from the job variables studied and the burnout.

\section{Method}

\section{Participants}

The sample was 250 immigrants from Latin America (41.3\%), Eastern Europe (26.3\%), Maghreb (27.5\%) and sub-Saharan Africa (4.9\%). All participants are working in unskilled jobs, construction, domestic service and caring for people or hotels (cleaning or kitchen help).

\section{Instruments}

It has developed a structured interview with closed questions on demographics, immigration-related data and data from the social area. For the measurement of job satisfaction has been used Job Satisfaction Scale proposed by Warr, Cook and Wall (1979), NTP 394. For the measurement of burnout using a spanish version (Gil Monte, 2002) of the Maslach Burnout Invenntory-General Survey (MBI-GS, Maslach, Jackson \& Leiter, 1996). The job characteristics are assessed by seven items, raising the following statement: "you think that your work has the following characteristics ..." (strict control, immobility, noise, repetitive tasks, long hours, boring work, lack of stability). Satisfaction with the amount of leisure time that allows their work-free time-is assessed with one item: "Your job allows you to be satisfied with the time off."

\section{Results}

The main results are: a) there are no significant differences by gender of the participants in burnout, or job satisfaction, b) there are significant differences by gender in job characteristics, resulting in higher levels of perception of noise and hours worked for the group of men, c) there are no differences according to whether the administrative situation, the shift, working hours, the contract and the level of training in the levels of job satisfaction and burnout. We get high and statistically significant correlations between job satisfaction for men and women $(p<.001)$.

\section{Discussion}

Regarding the sex differences reject the hypothesis of differences in job satisfaction and burnout, however, notes that women in worse conditions did not show a decrease in job satisfaction. The rest of variables proposed (origin, administrative status, shift, working hours, contract, and level of education) do not produce differences in burnout, or job satisfaction. The hypothesis on the relationship between job satisfaction and burnout is confirmed. It is necessary to highlight the importance of satisfaction seems to have the free time on job satisfaction. Second, the importance of promoting satisfaction with free time is enhanced by being a sample of immigrants, since in this case there is also evidence of how the use of leisure time is related to the acculturation process in new culture (Christenson, Zabriskie, Eggett \& Freeman, 2006; Stack \& Iwasaki, 2009), the latter variable could also be related to job satisfaction.

Another variable that predicts the two models proposed (job satisfaction in men and women) is the perception of job security. Finally, in the female sample found that tight control of tasks negatively predicts job satisfaction. This information does not appear in men. As limitations of this study indicate that following a cross-sectional design, so that you can observe the evolution of burnout or job satisfaction over time. Another limitation concerns the sample characteristics and is considered a single group of workers who have in common the fact that immigrants, however, have different jobs, but all low-skilled. Despite these limitations, highlights the dearth of studies focusing on job 
satisfaction and burnout among the immigrant population. In an ever changing society, the challenge is to learn to balance social responsibility - focus on cultural diversity, with economic efficiency.

\section{Referencias}

Achotegui, J. (2002). La depresión en los inmigrantes. Una perspectiva transcultural. Barcelona: Editorial Mayo.

Achotegui, J. (2004). Emigrar en situación extrema: el Síndrome del inmigrante con estrés crónico y múltiple (Síndrome de Ulises). Norte de Salud Mental, 21, 39-52.

Alonso, P. (2008). Estudio comparativo de la satisfacción laboral en el personal de administración. Revista de Psicología del Trabajo y de las Organizaciones, 24, 2540 .

Alvarez, B. y Miles, D. (2006). Husband's housework time: Does wives' paid employment make a difference? Investigaciones Económicas, 30, 5-31.

Aycan, Z. y Berry, J.W. (1996). Impact of employmentRelated Experiences on immigrants' Psychological WellBeing and Adaptation to Canada. Canadian Journal of Behavioural Science, 28, 240-251.

Berry, J.W. (1997). Immigration, acculturation and adaptation. Applied Psychology: An International Review, 46, 5-34.

Berry, J. W., Poortinga, Y. H., Segal, M. H. y Dansen, P. R. (1992). Cross-cultural psychology. Reseach and applications. Cambridge: University Press.

Bravo, R.M. (1993). El proceso de adaptación socio-cultural de los refugiados. III Jornadas de Psicología de la Intervención social, 3, 1275-1284. Madrid, noviembre 1991.

Bravo, Ma . J., Peiró, J. M. y Rodríguez, I. (1996). Satisfacción laboral. En J. M. Peiró y F. Prieto (Eds), Tratado de Psicología del Trabajo. Vol. I: la actividad laboral en su contexto (pp.347-394). Madrid. Síntesis.

Brief, A. P. (1998). Attitudes In and Around Organizations. Thousand Oaks. Sage.

Christenson, O., Zabriskie, R., Eggett, D. y Freeman, P. (2006). Family acculturation, family leisure involvement, and family functioning among Mexican-Americans. Journal of Leisure Research, 38, 475-495.

Claes, R. (1987). La centralidad del trabajo en la vida de los jóvenes. En J.M. Peiró y D. Moret (Dirs.) Socialización laboral y desempleo juvenil: La transición de la escuela al trabajo (pp. 81-100). Valencia: Nau Libres.

Clark, A. E. (1997). Job satisfaction and gender: why are women so happy at work? Labour Economics, 4, 341-372.

Cruz, A.C., Noriega, M. y Garduño, M.A. (2003). Trabajo remunerado, trabajo doméstico y salud. Las diferencias cualitativas y cuantitativas entre mujeres y varones. Caderno Saúde Pública, 19, 1129-1138.

Farley, T., Galves, A., Dickinson, M. y Díaz, M.J. (2005). Stress, doping and mental health: A comparison of Mexican immigrants, Mexican-Americans, and NonHispanic whites. Journal of Immigrant Health, 3, 213-220.
Forgionne, G. A. y Peeters, V. E. (1982). Differences in job motivation and satisfaction among female and male managers. Human Relations, 35, 101-118.

Gamero, C. (2004). Satisfacción laboral de los asalariados en España. Especial referencia a las diferencias por género. Cuadernos de Economía, 27, 109-146.

Gamero, C. (2007). Satisfacción laboral y tipo de contrato en España. Investigaciones Económicas, 31, 415-444.

Gamero, C. (2009). Valoración de las características de los empleos por nativos e inmigrantes: el enfoque de la satisfacción con la vida. Principios. Estudios de Economía Política, 14, 93-111.

García, M., Castellón, M., Albaladejo, B. y García, A. (1993). Relaciones entre Burnout, ambigüedad de rol y satisfacción laboral en el personal de Banca. Psicología del Trabajo y Organizaciones, 11, 17-26.

Garden, A.M. (1989). Burnout: the effect of psychological type on research findings. Journal of Occupational Psychology, 62, 223-234.

Gil-Monte, P.R. (1999). Validez factorial del Maslach Burnout Inventory en una muestra multiocupacional. Psicothema, 11, 679-689.

González, M.T. y Landero, R. (2007). Factor structure of perceived stress scale (PSS), in a sample from Mexico. The Spanish Journal of Psychology, 1, 199-206.

Green, D.E., Walkey, F.H. y Taylor, J.W. (1991). The threefactor structure of the Maslach burnout inventory. Journal of Science Behaviour and Personality, 6, 453472.

Haworth, J.T. (1997). Work, leisure and well-being. London: Routledge.

Haworth, J.T. y Veal, A.J. (2004). Work and Leisure. London: Routledge.

Hoppock, R. (1935). Job Satisfaction. New York: Harper Ed.

Linz, S. J. (2003). Job satisfaction among Russian workers. Journal of Manpower, 24, 626-645.

Locke, E.A. (1976). The nature and causes of job satisfaction. En M.D. Dunnette (Ed.), Handbook of Industrial and Organizational Psychology (pp. 1297-1349). Chicago, IL: Rand McNally.

López-Araújo, B., Osca, A. y Peiró, J.M. (2007). El papel modulador de la implicación con el trabajo en la relación entre el estrés y la satisfacción laboral. Psicothema, 19, 81-87.

Maslach, C. y Jackson, S. (1986). Maslach Burnout Inventory Manual. Palo alto, CA: Consulting Psychologists.

Maslach, C., Schaufeli, W. B. y Leiter, M. P. (2001). Job burnout. Annual Review of Psychology, 52, 397-422.

Miller, K., Ellis, B., Soc, E. y Lyles, J. (1990). An integrated model of communication, stress, and burnout in the workplace. Communication Research, 17, 300-326.

Muñoz, A. (1990). Satisfacción e insatisfacción en el trabajo. Tesis doctoral. Universidad Complutense de Madrid. España.

Newbury, D. y Kamali, F. (2001). Psychological stress, anxiety, depression, job satisfaction and personality characteristics in preregistration house officers. Post Graduate Medical Journal, 77, 109-111. 
Oshagbemi, T. (2000). How satisfied are academics with their primary tasks of teaching, research and administration and management. International Journal of Sustainability in Higher Education, 1, 124-136.

Ovejero, A. (2006). El mobbing o acoso psicológico en el trabajo: una perspectiva psicosocial. Revista de Psicología del Trabajo y de las Organizaciones, 22, 101121.

Patiño, C. y Kirchner, T. (2009). Estrés y calidad de vida en inmigrantes latinoamericanos en Barcelona. Ansiedad y Estrés, 15, 49-66.

Peiró, J.M. (1984). Psicología de la organización. Madrid: UNED.

Porthé, V., Amable, M. y Benach. J. (2007). La precariedad laboral y la salud de los inmigrantes en España: ¿qué sabemos y qué deberíamos saber?. Archivos de Prevención Riesgos Laborales, 10, 34-39.

Prada, M.A., Actis, W. y Pereda, C. (2000). Perspectiva laboral de la inmigración en España. Documentación Social: Revista de Estudios Sociales y Sociología Aplicada, 121, 91-110.

Pumares-Fernández, P., García, A. y Asensio, A. (2006). La movilidad laboral y geográfica de la población extranjera en España. Madrid: Ministerio de Trabajo y Asuntos Sociales (OPIM).

Ramos, P.J. y García-Izquierdo, A.L. (2007). La medida del síndrome de Ulises. Ansiedad y Estrés, 13, 253-268.

Renaud, S. (2002). Rethinking the union membership/job satisfaction relationship. Some empirical evidence in Canada. Internacional Journal of Manpower, 23, 137-150.

Rinken, S. (2008). Condiciones laborales y calidad de vida de los inmigrantes en Andalucía. Calidad de Vida UFLO, 1, 52-66.

Ritsner, M. y Ponizovsky, A. (1999). Psychological distress through immigration: The two-phase temporal pattern. International Journal of Social Psychiatry, 45, 125-139.

Salanova, M., Gracia, F. J. y Peiró, J. M. (1996). Significado del trabajo y valores laborales. En J. M. Peiró y F. Prieto (Eds), Tratado de Psicología del Trabajo. Vol. II: Aspectos psicosociales del trabajo (pp.35-63). Madrid. Síntesis.

Salanova, M. y Llorens, S. (2008). Estado actual y retos futuros en el estudio del burnout. Papeles del Psicólogo, 29, 59-67.

Salanova, M., Prieto, F. y Peiró, J.M. (1993). El significado del trabajo: Una revisión de la literatura. En J.M. Peiró, F. Prieto, M.J. Bravo, P. Ripoll, I. Rodríguez, P. Hontangas y M. Salanova (Eds.): Los jóvenes ante el primer empleo: el significado del trabajo y su medida. Valencia: Nau Llibres.

Sánchez, S.M., Fuentes, F.J. y Artacho, C. (2007). La perspectiva de género en el análisis de la satisfacción laboral: una aplicación empírica mediante modelos logit y probit. Cuadernos de Gestión, 7, 55-67.
Sánchez-Herrero, S. (2008). La importancia de la perspectiva de género en la psicología del ocio. Anales de Psicología, 24, 67-76.

Sánchez-López, M. P. (1997). El estilo psicológico como estudio de la diversidad humana: un ejemplo basado en los estilos de vida. Revista de Psicología, 15, 223-252.

Sánchez-López, Ma P. y Aparicio, M. (2001). Estilo de vida: avances en su medida y sus relaciones con otros conceptos psicológicos. Revista de Psicología, 19, 5-26.

Sanz de Galdeano, A. (2001). Gender differences in job satisfaction and labour market participation: UK evidence form propensity score. Presentado en 13th EALE Annual Conference, 13-16 sept., Jyväskylä, Finlandia.

Schaufeli, W. y Enzmann, D. (1998). The burnout companion to study and practice. A critical analysis. London: Francis y Taylor.

Sloane, P.J. y Williams, H. (2000). Job satisfaction, comparison earning and gender. Labour, 14, 473-501.

Solé, C. (2001). El impacto de la inmigración en la economía y en la sociedad receptora. Barcelona: Antrophos.

Stack, J.C.A. y Iwasaki, Y. (2009). The role of leisure in adaptation processes among Afghan refugees who have immigrated to Winnipeg, Canada. Leisure Studies, 28, 239-259.

Taylor, S.J. y Bogdan, R. (1986). Introducción a los métodos cualitativos de investigación. La búsqueda de significados. Buenos Aires y Barcelona: Editorial Paidós.

Topa, G., Fernández, I. y Lisbona, A. (2005). Ruptura de contrato psicológico y burnout en equipos de intervención en emergencias y catástrofes. Ansiedad y Estrés, 11, 265-279.

Vallejo, M. (2009). Satisfacción Vital en el proceso de aculturación de inmigrantes, propuesta de un modelo. Tesis doctoral no publicada. Departamento de Psicología Social, Antropología Social, Trabajo Social y Servicios Sociales. Universidad de Málaga.

Virtanen, M., Kivimaki, M., Elovainio, M, Vahtera, J. y Ferrie, J. (2003). From insecure to secure employment: changes in work, health, health relate behaviors and sickness absence. Occupational Environmental Medicine, 60, 948-953.

Ward, M. E. y Sloane, P. J. (2000). Non-pecuniary advantages versus pecuniary disadvantages: job satisfaction among male and female academics in Scottish universities. Scottish Journal of Political Economy, 47, 273-303.

Warr, P., Cook, J. y Wall, T. (1979). Scales for the measurement of some work attitudes and aspects of psychological well-being. Journal of Occupational Psychology, 52, 129-148.

Wooden, M. y Warren, D. (2004). Nonstandard employement and job satisfaction: Evidence from the HILDA survey. The Journal of Industrial Relations, 46, 275297. 